\title{
Motley String or from 10 to 4
}

\section{Matveev GY*}

PhD, Algebraic Number Theory, McGill University in Montreal, Canada

\section{Introduction}

New compactification mechanism of String theory is proposed. Applied to Superstring theory it explains quarks fractional charges and quark/lepton generations of the Standard Model. Theory also explains Regge slope values, quarks mixing and neutrinos oscillations. Also it offers explanation for "Dark matter" puzzles of modern astrophysics.

Superstring theory [1] is considered by many High-Energy physicists as a good candidate for Theory of Everything since it allows for formulation of all fundamental forces of nature (Electromagnetic, Strong, Weak and Gravitational) as modes of excitations of strings.

However, it turns out that String theory can only be consistently formulated either in 10 (Superstring) or 26 (Bosonic and Heterotic strings) dimensions $[1,2]$.

The original idea of formulating physical theory in higher than 4 dimensional space-time goes back to 1914 when physicist from Helsingfors Gunnar Nordstrom introduced extra space dimension to include electromagnetism in his theory of gravitation [3].

In 1919 Theodor Kaluza realized that solving Einstein's equations (published one year after Nordström's theory) for general relativity using five dimensions automatically produces Maxwell's equations for electromagnetism. He published his discoveries in 1921 after discussions with Einstein [4].

At the same time most of observational material tells us that we live in 4 dimensional space-time. To solve this "compactification" problem two different approaches were suggested: Kaluza-Klein and Calabi-Yau.

In 1926 O. Klein proposed [5] that extra spacial dimension in Kaluza theory got curled up in a circle of small radius (aka "compactified"). Same idea applied to Superstring theory means that some spacial dimensions (6) are compactified, while others (3) are not, which leaves unanswered question why some dimensions are better then others. Also it implies the existence of standing waves in compactified dimensions (so called Kaluza-Klein tower) which are not observed experimentally.

Calabi-Yau solutions suggest that 6 spatial extra dimensions are compactified as complex 3 dimensional manifolds (with 6 real dimensions) [6,7]. These are reaching and sophisticated mathematical models based on existence of compact Kahler manifolds with vanishing Ricci curvature [6]. There are two alternatives here to choose from: one is that "hidden" spacial dimensions are so small that they cannot be observed today. Another (popular in D-brane theories) is exactly the opposite - extra dimensions are large, but we are confined to a small subset on which it intersects with D-brane $[8,9]$.

To avoid all those inconsistencies present in both approaches to compactification we propose "Motley string" model, which treats all spacial dimensions equally and complies with known experimental material.

\section{True Colors of Strings}

First we formulate two Postulates:

1. Postulate 1: Every spacial dimension of String has a unique intrinsic property which we call "color".
2. Postulate 2: There is force between spacial dimensions of string such that it makes dimensions of complementary colors (Red, Green $\left.B l u e_{i}\right)$ in-teract and unite in a colorless threads perceived as observable dimensions.

String state at very high energies (early universe, Planck length about $10^{-33} \mathrm{~cm}$ ) is such that all spacial dimensions of the String are in a free state similar to quark-gluon plasma of Quantum Chromo Dynamics [10] (Figure 1).

At lower energies (modern universe) strong "color" force becomes dominant and makes String's complimentary, or using classical optics term "additive" spacial dimensions $\left(\right.$ Red $_{i}$, Green $_{i}$, Blue $\left._{i}\right)$ interact to form 3 threads (in case of 9+1 dimensional Superstring), which appear to be colorless from distances larger than size of baryons (proton, neutron, etc). Spacial dimensions of additive "colors" are "glued" together due to Color Force. As a result humans perceive the original $(9+1)$ dimensional String as $3+1$ dimensional space time! See Figure 2 below.

Outside of Planck energy scale strings spacial dimensions are confined in 3 colorless observable threads.

Since in our model all spacial dimensions are treated uniformly we avoid questions like "why some spacial dimensions are compactified while others are not".

Also there are no standing waves in curved dimensions of Klein compactification and therefore no extra mass values (aka Kaluza-Klein tower).

Equally important there is no need for Calabi-Yau and somewhat artificial "large extra dimensions" models invented to explain "unseen" spacial dimensions [9].

String tension $T$ is related to the Fundamental length of String $l$ via:

$l^{2=} 1 /(\pi-T)$

String tension $T$ is also related to so called Regge slope $\alpha^{\prime}$ (of QCD) which could be experimentally measured via:

$T=1 /\left(2 \pi-\alpha^{\prime}\right)[1]$

Regge slope $\alpha^{\prime}$ is in turn related to particle spin $J$ and mass $M$ via:

$\alpha^{\prime}=J / M^{2}$

and is measured to be around $1 \mathrm{GeV}^{-2}$.

Major textbook on String theory "Superstring theory" is quite vague on string Tension $T$, the key element of string theory. On page one it is mentioned that Regge slope is about $1 \mathrm{GeV}-2$ [1].

But closer look at Regge slope reveals something VERY interesting and ALL important: leading Regge trajectories of (quark - anti quark)

*Corresponding author: Matveev GY, PhD, Algebraic Number Theory, McGill University in Montreal, Canada, Tel: +1 514-398-4455; E-mail: george@matveev.se

Received May 29, 2018; Accepted June 29, 2018; Published July 06, 2018

Citation: Matveev GY (2018) Motley String or from 10 to 4. J Laser Opt Photonics 5: 190. doi: 10.4172/2469-410X.1000190

Copyright: (c) 2018 Matveev GY. This is an open-access article distributed unde the terms of the Creative Commons Attribution License, which permits unrestricted use, distribution, and reproduction in any medium, provided the original author and source are credited. 


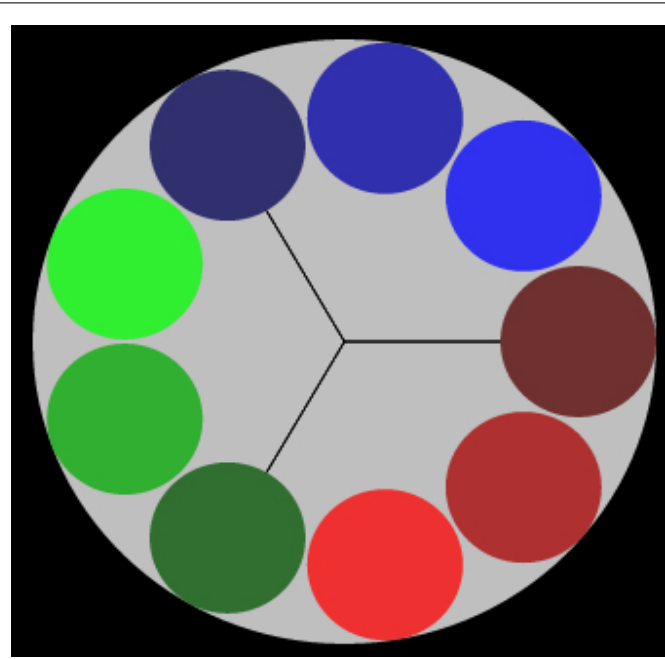

Figure 1: Motley string at Plank scale.

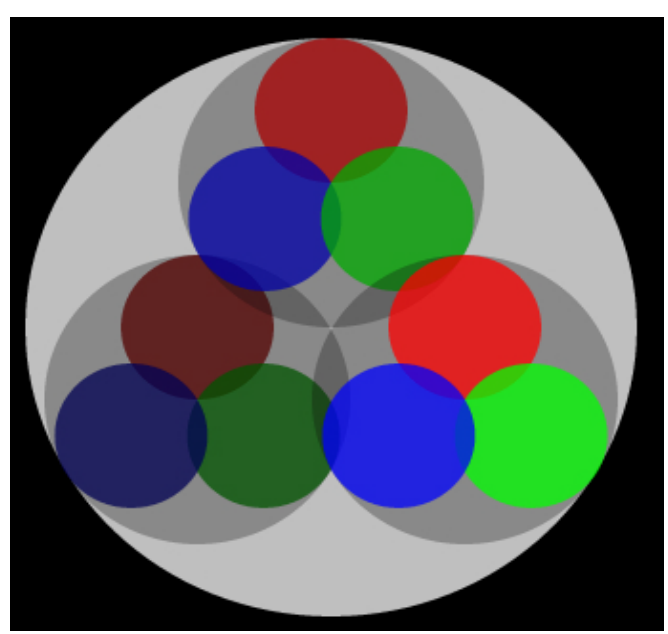

Figure 2: Motley string in Modern Cold Universe

mesons are quite different for mesons built of different quark - anti quark pairs [11]!

Which is exactly what one would expect given different composition of strings ( Red $_{i}$, Green ${ }_{i}$ Blue $e_{i}$ ) of tensions $T_{i}$ for different quarks making up mesons.

Nambu-Goto string with massive spinning quarks model shows that Regge slopes for different (quark-anti quark) mesons are quite different!, which contains both experimental values and computational results and references [12].

This fact can be seen as the first and most direct confirmation of Motley String theory! Both experimentally and by different computational models of (quark-anti quark) mesons [13].

\section{Applications to Standard Model and Cos-mology}

Excitation (e.g., soliton) on one original color dimension of a colorless thread could be considered a quark of charge $1 / 3$, while excitation on 2 color dimensions of a colorless thread could be perceived as a quark of charge $2 / 3$. Total number of different modes of excitations on 3 color dimensions on each thread equals 6 ( 3 single +3 pairs) and thus we arrive at 6 flavors of quarks (up, down, charm, strange, top, bottom) known from Standard Model [14].

In a similar way, different types of excitations not related to "color" which live on 3 colorless observable dimensions result in 6 known types of leptons (electron, muon, tau lepton, and their corresponding neutrinos $v$ ) [14].

Since color force is much stronger than Electromagnetic and Weak forces, energy required to produce excitation on String color components/dimensions is much higher than energy required producing excitation of observable colorless dimensions. And that explains why quark masses (which make up mesons and baryons) are generally higher than masses of leptons (electron, muon, etc.).

Interestingly, Motley String model could be easily applied to Bosonic String formulated in 26 dimensions $\left(26=1+5^{2}\right)$ and Heterotic String (combination of left-moving excitations of Bosonic string and right-moving modes of Superstring).

At the same time Motley String theory also offers plausible explanation for "Dark Matter puzzle" present in modern cosmology, which basically states that about $95 \%$ of total observable matter of the Universe is made of unseen "dark" matter/energy [15].

According to data available on Particle Data Group web site [16] we have for Up (+2/3) and Down (-1/3) quark masses:

Mass of U quark: $\sim 2.3 \mathrm{MeV}$

Mass of D quark: 4.8 MeV

While at the same time masses of Neutron and Proton (composed of Up and Down quarks) are:

\section{Mass of Neutron $(\mathrm{U}+\mathrm{D}+\mathrm{D}): \sim 1008 \mathrm{MeV}$ \\ Mass of Proton $(\mathrm{U}+\mathrm{U}+\mathrm{D}): \sim 940 \mathrm{MeV}$}

Which means that most ( 95\%) of baryon masses come from so called "binding energy" (or quark-gluon plasma), not from individual masses of quarks making up hadron.

One may say that Proton and Neutron masses are mostly a result of "collective binding energy" of those up and down quarks that make them up!

Much the same like hadrons masses, most of the Universe observable mass comes from energy related to Motley String spacial dimensions, compactified because of Color Force, and therefore not seen (aka "Dark matter").

Which means that "Dark matter/energy problem" may in fact be seen as experimental confirmation of Motley String theory.

Careful re-calculations (e.g., using Lattice QCD methods) of quark/hadron and lepton masses based on our Motley String model could possibly shed some light on "dark matter" mystery of modern astrophysics. Very close proximity of those numbers (95 $\sim 96 \%)$ seems to indicate that compactification of String's spacial dimensions due to Color Force may be the explanation of the "Dark energy/matter" [17].

Motley String theory also offers alternative to Higgs mechanism of mass generation for particles and therefore explains how neutrinos get their mass from modes of excitations of compactified spacial dimensions of Motley String. 
This in turn makes hunting of hypothetical Right-handed neutrinos (required by Higgs mechanism) not necessary because neutrinos are known to be Left-handed (to take part in Weak interactions), and their mass origin is now explained by Motley String theory.

\section{Motley String on Quark mixing and Neu-trino Oscilla- tions}

For quarks of charge $-1 / 3(d, s, b)$ there are following three combinations of colors/flavors on single colorless spacial dimensions of Motley String:

1. $\left(\right.$ Red $_{1}$, Green $_{1}$, Blue $\left._{1}\right)$

2. $\left(\right.$ Red $_{2}$, Green $_{2}$, Blue $\left._{2}\right)$

3. $\left(\right.$ Red $_{3}$, Green $_{3}$, Blue $\left._{3}\right)$

And for quarks of charge $+2 / 3(u, c, t)$ we have another 3 possible combinations of colors/flavors on two spacial colorless dimensions of Motley String:

$$
\begin{aligned}
& \text { 1. }\left(\text { Red }_{1}, \text { Green }_{1}, \text { Blue }_{1}\right)+\left(\text { Red }_{2}, \text { Green }_{2}, \text { Blue }_{2}\right) \\
& \text { 2. }\left(\text { Red }_{1}, \text { Green }_{1}, \text { Blue }_{1}\right)+\left(\text { Red }_{3}, \text { Green }_{3}, \text { Blue }_{3}\right) \\
& \text { 3. }\left(\text { Red }_{2}, \text { Green }_{2}, \text { Blue }_{2}\right)+\left(\text { Red }_{3}, \text { Green }_{3}, \text { Blue }_{3}\right)
\end{aligned}
$$

On the other hand Gluons are known to have and transmit "color charge" between quarks.

Therefore one might think that "quark mixing" between six known types of quarks above is related to exchange of Gluons between String's colorless threads. And so called "quark mixing angle" introduced by Nicola Cabibbo in 1963 in an attempt to explain quark mixing [14,18] is a direct result of ex-change of Gluons between String's colorless threads (aka observable spacial dimensions).

In a similar fashion we have 3 different types of excitations on single colorless spacial dimensions for electron, muon and tau leptons, as well as 3 more modes of excitations on two spacial colorless dimensions for three different types of neutrinos (electron, muon and tau neutrinos).

Now if we replace QCD bosons (Gluons) with Weak force bosons ( $\mathrm{W}$ and $\mathrm{Z}$ ) we get exactly the same mechanism working and mixing (aka oscillating) both quarks and neutrinos!

Therefore we have to conclude that Motley String theory provides expla-nation for both "quark mixing" and neutrino oscillations detected by 3 major experiments (South Dacota, Sudbury and SuperKamiokade) [19].

\section{Conclusion}

Motley String model and idea of String's "colorful" spacial dimensions introduced in this article offers consistent and uniform approach to compactification problem present in all String models (Superstring, Bosonic, Heterotic).

It eliminates inconsistencies of compactification mechanisms proposed earlier (Kaluza-Klein, Calabi-Yau manifolds, etc.).
Also it solves several major problems present in the Standard Model:

1. Number of particles (6 quarks and 6 leptons) in the Standard Model.

2. Fractional charges of quarks and quark/gluon confinement.

3. The link between High-dimensional String theories and observable 4-dimensional world.

4. Alternative to Higgs mechanism for particles mass generation and thus explains neutrino's mass and experimentally observed neutrinos oscillations.

5. Single mechanism for both quark mixing and neutrino oscillations.

6. Solution for "dark matter/energy" problem of modern astrophysics.

\section{References}

1. Green MB, Schwarz JH, Witten E (1987) Superstring Theory. Oxford University Press, New York.

2. Green MB, Schwarz JH (1984) Anomaly Cancellations in Su-persymmetric D=10 Gauge Theory and Superstring Theory. Physics Letters B 149: 117-122.

3. Nordstrom G About (1914) the possibility of uniting the electromagnetic field and the gravitational field. In: Phys J 15: 504-506.

4. Kaluza T (1921) On the Unification Problem in Physics. physics.hist-ph, pp 966-972.

5. Klein O (1926) Quantum Theory and Five Dimensional Theory of Relativity magazine for physics 37: 895-906.

6. Calabi E, Chen XX (1954) The Space of Kähler metrics (II). math.DG, pp: 1-16.

7. Green BR (1987) String Theory On Calabi-Yau Manifolds. Hep-th, pp: 1-150.

8. Polchinski J (1997) TASI Lectures on D-branes. Hep-th, pp: 1-63.

9. Brandenberger R, Vafa C 1989) Superstrings in the early universe. Nucl Phys B 316: 391-410.

10. Greiner W, Schafer A (1994) Quantum Chromodynamics. Springer 9.

11. Veneziano G (1968) Construction of a crossing-symmetric, Reggeon-behaved amplitude for linearly rising trajectories. Nuovo Cemento 57A: 190-197.

12. Soloviev LD (1998) Relativistic quantum model of confinement and the cur-rent quark masses. Phys.Rev pp-1-6.

13. DiVecchia $P$ (2008) The Birth of String Theory Lect Notes Phys 737: 59-118.

14. Halzen F, Martin A (1984) Quarks \& Leptons An Introductory Course in Modern Particle Physics, John Wiley \& Sons.

15. https://en.wikipedia.org/wiki/Dark_matter

16. http://pdg.lbl.gov/

17. Wiczek F (2000) QCD Made Simple. Phys Today 53: 22.

18. Matveev VA, Tavkhelidze AN (2006) The Quantum Number Color, Colored Quarks and Qcd. Report presented at the 99th Session of the JINR Scientific Council, Dubna, 19-20 January.

19. Garcia MCG, Maltonic M, Schwetz T (2016) Global analyses of neutrino oscillation experiments. Nucl Phys B 908: 199-217. 